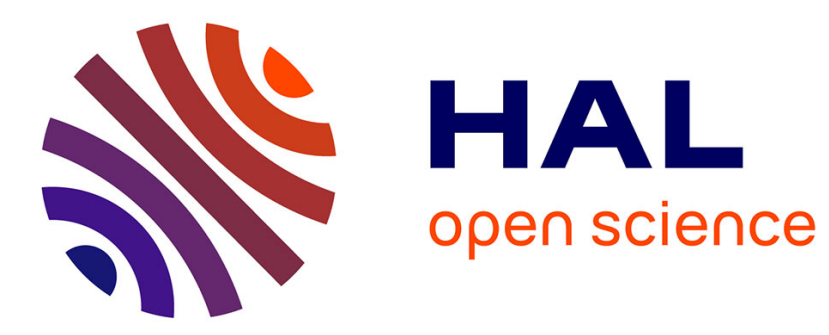

\title{
On the Use of a Complex Frequency for the Description of Thermoacoustic Engines
}

Matthieu Guédra, Guillaume Penelet

\section{To cite this version:}

Matthieu Guédra, Guillaume Penelet. On the Use of a Complex Frequency for the Description of Thermoacoustic Engines. Acta Acustica united with Acustica, 2012, 98 (2), pp.232-241. hal-02057411

HAL Id: hal-02057411

https://hal-univ-lemans.archives-ouvertes.fr/hal-02057411

Submitted on 5 Mar 2019

HAL is a multi-disciplinary open access archive for the deposit and dissemination of scientific research documents, whether they are published or not. The documents may come from teaching and research institutions in France or abroad, or from public or private research centers.
L'archive ouverte pluridisciplinaire HAL, est destinée au dépôt et à la diffusion de documents scientifiques de niveau recherche, publiés ou non, émanant des établissements d'enseignement et de recherche français ou étrangers, des laboratoires publics ou privés. 


\title{
On the use of a complex frequency for the description of thermoacoustic engines
}

\author{
M.Guedra ${ }^{a, *}$, G. Penelet ${ }^{a}$. \\ ${ }^{a}$ Laboratoire d'Acoustique de l'Université du Maine, UMR CNRS 6613, Avenue Olivier Messiaen, 72085 Le \\ Mans Cedex 9, FRANCE \\ *Corresponding author, E-mail: matthieu.guedra.etu@univ-lemans.fr, fax.: +33-2-43-83-35-20
}

\begin{abstract}
In this paper, a formulation is proposed to describe the process of thermoacoustic amplification in thermoacoustic engines. This formulation is based on the introduction of a complex frequency which is calculated from the transfer matrices of the thermoacoustic core and its surrounding components. The real part of this complex frequency represents the frequency of self-sustained acoustic oscillations, while its imaginary part characterizes the amplification/attenuation of the wave due to the thermoacoustic process. This formalism can be applied to any type of thermoacoustic engine including stack-based or regenerator-based systems as well as straight, closed loop or coaxial duct geometries. It can be applied to the calculation of the threshold of thermoacoustic instability, but it is also well-suited for the description of the transient regime of wave amplitude growth and saturation due to non linear processes. All of the above mentioned aspects are described in this paper.
\end{abstract}

PACS numbers: 43.35.Ud 


\section{Introduction}

Thermoacoustic engines belong to a type of heat engines in which acoustic work is produced by exploiting the temperature gradient between a hot source and a cold sink [1, 2]. Typical arrangements of thermoacoustic engines are shown in Fig. 1. It consists basically of an acoustic resonator partially filled with a piece of an opencell porous material, often referred to as a stack or a regenerator. An important temperature gradient is imposed along this stack/regenerator, so that above a critical temperature gradient, acoustic modes of the resonator can become unstable and the thermoacoustic process results in the onset of self-sustained, large amplitude acoustic oscillations. Such kind of engines have been extensively studied in the past decades [3], leading to a deeper understanding of their operation and to the building of a few devices such as thermoacoustically driven thermoacoustic refrigerators or thermoelectric generators. These engines have interesting features inherent to the absence of moving parts (pistons and crankshafts) which can be advantageously used for industrial applications at moderate power densities (typically up to a few kilowatts). It is however worth noting that the design of thermoacoustic engines is a tedious task which comprises an important part of uncertainties, because the operation of these engines is by nature nonlinear, and because the existing efficient prototypes include various elements like flow straighteners, tapered tubes, membranes or jet pumps which are difficult to model accurately.

Many researchers use the freely available software package called DeltaEC developed at Los Alamos National Laboratory [4] for the design of thermoacoustic systems. This computer code is a very powerful tool which is mainly based on the linear (and weakly nonlinear) thermoacoustic theory in the frequency domain. Besides the limitations of this computer code for large acoustic amplitudes requiring proper account of nonlinear effects, another of its characteristics is that it is expressed in the Fourier domain, so that it describes steady state conditions : the steady-state acoustic pressure amplitude is obtained from a temperature field which itself is controlled by the acoustic field due to acoustically induced heat transport. The multi-parameter shooting method which is employed in this computer code is well suited for the prediction of an equilibrium state above the threshold of thermoacoustic instability. However, it is not primarily devoted to the determination of the 


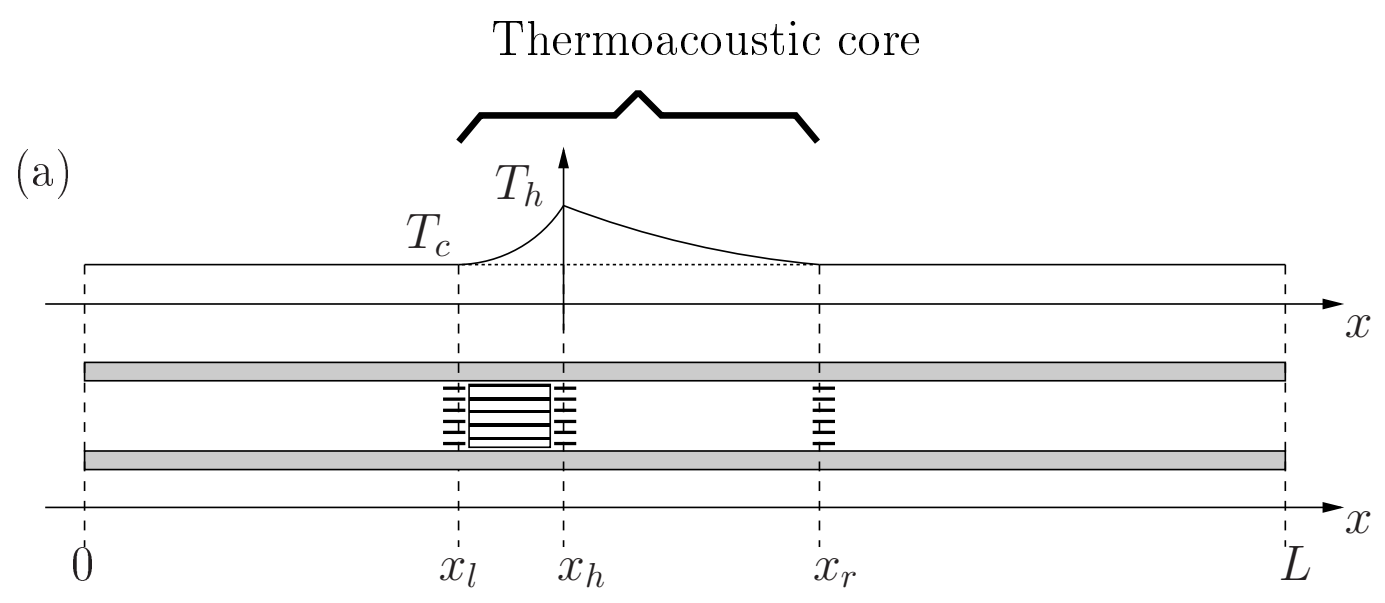

(b)

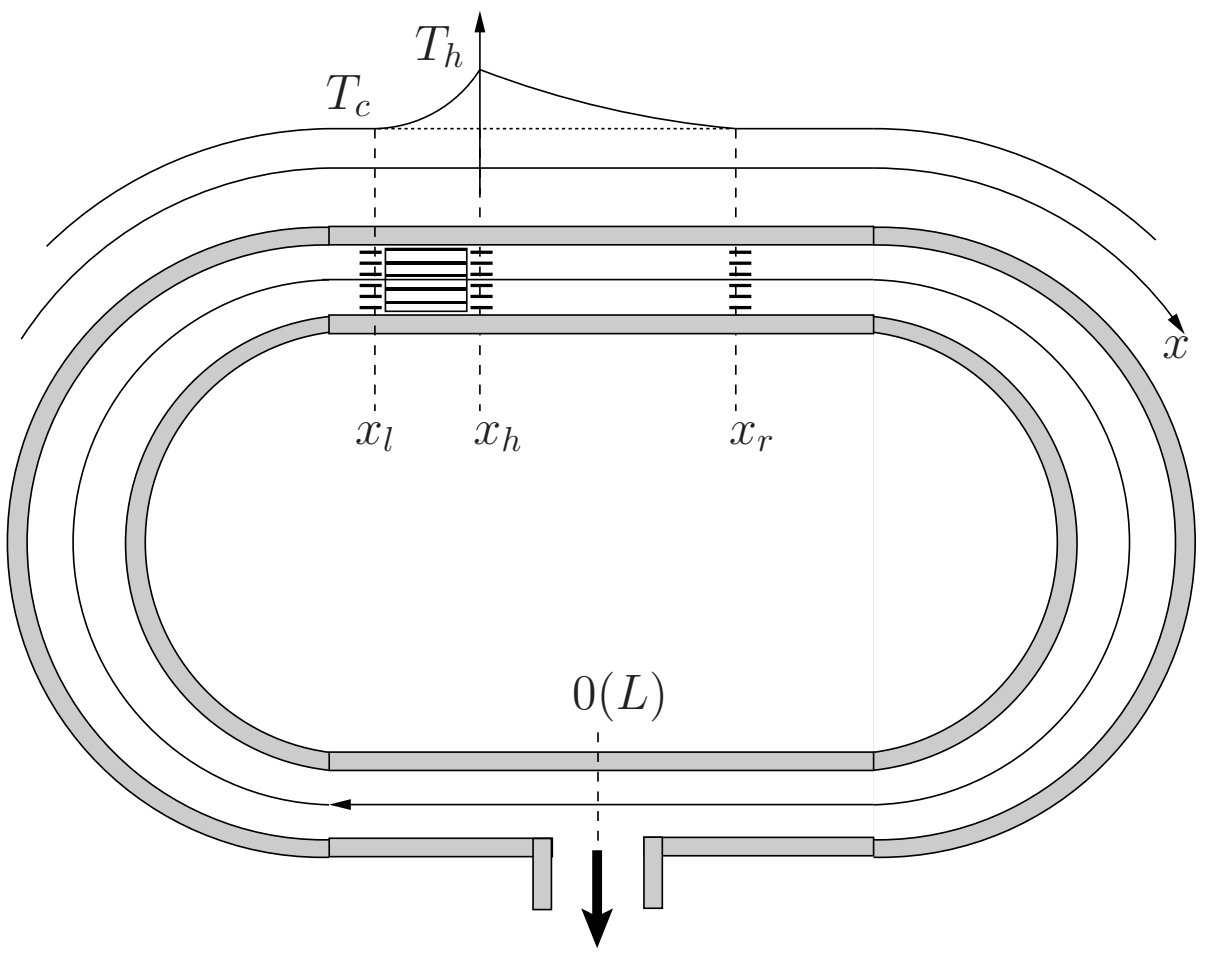

to secondary acoustic load

Figure 1: Simplified drawings of a standing wave engine (a) and a travelling wave loop engine (b), possibly coupled with a secondary acoustic load.

threshold condition itself (i.e. the required external thermal action above which thermoacoustic oscillations begin to grow up with time). Moreover, under some circumstances, the transient process leading to the steady state sound should be considered, and the approach used in DeltaEC then becomes unsuitable. This is notably the 
case when the engine is operated slightly above the threshold of thermoacoustic instability, where complicated effects may be observed. For example, the existence of a hysteretic loop $[5,6]$ in the onset and damping of the engine, or the periodic switch on/off of thermoacoustic instability $[7,8]$ have been reported for both standing and travelling wave engines. In such situations, the fixed external thermal action on the system does not correspond to a unique steady state solution for the acoustic pressure amplitude.

Though useful design tools are nowadays available, an accurate description of thermoacoustic engines is still needed, and an important research effort has been devoted to the description of the onset of thermoacoustic instability and to its saturation by nonlinear effects. Various analytical $[9,10,11,12]$ and numerical models $[13,14]$ have been proposed in the literature, which are yet limited to the description of simple thermoacoustic devices of particular geometry. In this context, the aim of this paper is to propose a general modelling approach which is mainly based on the transfer matrices formalism. As in previous analytical works $[9,12]$ the model presented in this paper takes advantage of the significant difference between the instability time scale and the period of acoustic oscillations, which is exploited here by the introduction of a complex frequency, sometimes used for the treatment of transient oscillatory motions (note that the introduction of complex frequency has already been proposed in a conference by J.E. Parker et al. [15] to treat thermoacoustic oscillations, and also in a similar network approach by Q. Tu et al. [16]). Depending on its sign, the imaginary part of this complex frequency represents an amplification or an attenuation coefficient, which is calculated from the linear thermoacoustic theory applied to the thermoacoustic system under consideration. The analytical treatment presented here is necessarily based on substantial approximations but, as will be discussed in this paper, it is well suited to carry out extensive parametric studies of both the transient and steady states. Moreover, this model has some interesting similarities with the computer code DeltaEC in the sense that it consists of a multiport network approach which is well-suited for the description of complicated acoustic networks including thermoacoustic cores, ducts with constant or varying cross-sections, grids, membranes, T-junctions etc ... The works presented in this paper basically consist of a generalization of previous works $[10,17]$ and its main 
originality is thus primarily to propose to the reader a rather simple modelling of any kind of thermoacoustic engine in order to determine its onset conditions or to describe the transient regime leading to steady state sound in the frame of weakly nonlinear theory.

In section 2 the multiport network modelling of thermoacoustic engines is presented, which leads to the analytical expression of the complex frequency from the transfer matrices of the thermoacoustic core and its surrounding components. In section 3, this formalism is applied to the determination of the conditions corresponding to the onset of thermoacoustic instability in the cases of a standing wave engine and of a closedloop travelling wave engine. Section 4 is devoted to the description of basic concepts concerning the use of this approach to study the transient regime leading to steady state sound (or to more complicated dynamic behaviors of the thermoacoustic oscillator) in thermoacoustic systems.

\section{Theory}

Thermoacoustic engines are generally made up of a duct network inside which the thermoacoustic core is inserted. The term "thermoacoustic core" refers here to the heterogeneously heated part of the device in which the amplification of acoustic waves operates: it is basically composed of an open cell porous material - referred to as the stack $\left(\delta_{\kappa} \sim r\right)$ or the regenerator $\left(\delta_{\kappa}>>r\right)$ depending on the value of the average radius $r$ of its pores relative to the thickness $\delta_{\kappa}$ of the acoustic thermal boundary layer - along which a temperature gradient is imposed using heat exchangers. As illustrated in Fig. 1, the great variety of thermoacoustic engines can be schematically separated into two different classes. The first class of engines (Fig. 1-(a)) refers to some conventional waveguide arrangement ensuring the resonance of a gas column. Among this class of engines are the stack-based standing wave engines which were extensively studied during the past decades, but also the cryogenic devices where Taconis oscillations may occur [1]. The second class of engines (Fig. 1-(b)) refers to some waveguide arrangements where a closed-loop path exists, allowing the development of travelling acoustic waves running along the loop. Among this class of engines are the stack-based travelling wave engine first studied 
by Yazaki et al. [18], the thermoacoustic-Stirling heat engine [19] first successfully designed by Backhaus et al. [20], the regenerator-based co-axial engines [21] where the feedback loop is formed by locating a small diameter thermoacoustic core into a larger diameter waveguide, and also as a matter of interest some kinds of free-piston Stirling engines.

Whatever the specific geometry of the engine under consideration, all of these devices use the fact that when a strong temperature gradient is imposed along the stack/regenerator, part of the heat supplied is converted into acoustic work inside the stack/regenerator. This thermoacoustic amplification process results in the onset of self-sustained, large amplitude acoustic waves oscillating at the frequency of the most unstable acoustic mode of the complete device. In the following, the onset of this thermoacoustic instability will be described by the introduction of a complex frequency, the real part of which describes the frequency of acoustic oscillations and the imaginary part of which describes the wave amplitude growth or attenuation. The analytical treatment presented here can be applied to any kind of thermoacoustic engine (and also to free piston Stirling engines), but it is convenient here for clarity to separate the cases where there exists or not a closed loop path for the acoustic waves. For the sake of simplicity, the first class of engine will be referred to as "standing wave" engine, while the second one will be referred to as "travelling wave" engine. The description of the acoustic field will be operated in the frequency domain in the frame of the linear approximation. Assuming that harmonic plane waves are propagating along the centerline of the ducts, the acoustic pressure $p(x, t)$ and acoustic volume velocity $u(x, t)$ are written as

$$
\zeta(x, t)=\Re\left(\widetilde{\zeta}(x) e^{-j \omega t}\right)
$$

where $\zeta$ may be either $p$ or $u, \tilde{\zeta}$ denotes the complex amplitude of $\zeta, \Re()$ denotes the real part of a complex number, and $x$ denotes the position along the duct axis (see Fig 1).

As shown in Fig. 1, the apparatus consists of a thermoacoustic core connected at both sides to straight (or curved) ducts. The propagation of acoustic waves through the thermoacoustic core is described as an acoustical 
two-port relating the complex amplitudes of acoustic pressure and volume velocity at both sides:

$$
\begin{aligned}
&\left(\begin{array}{c}
\widetilde{p}\left(x_{r}\right) \\
\widetilde{u}\left(x_{r}\right)
\end{array}\right)=\left(\begin{array}{cc}
\mathbf{T}_{\mathbf{p p}} & \mathbf{T}_{\mathbf{p u}} \\
\mathbf{T}_{\mathbf{u p}} & \mathbf{T}_{\mathbf{u u}}
\end{array}\right) \times\left(\begin{array}{c}
\widetilde{p}\left(x_{l}\right) \\
\widetilde{u}\left(x_{l}\right)
\end{array}\right), \\
&=\quad \mathbf{T}_{\mathbf{T C}} \times\left(\begin{array}{c}
\widetilde{p}\left(x_{l}\right) \\
\widetilde{u}\left(x_{l}\right)
\end{array}\right) .
\end{aligned}
$$

The transfer matrix of the thermoacoustic core, $\mathbf{T}_{\mathbf{T C}}$, depends on the geometrical and thermophysical properties of its components. It also depends on the temperature distribution $T_{m}(x)$ along the stack $\left(x \in\left[x_{l}, x_{h}\right]\right)$ and the thermal buffer tube $\left(x \in\left[x_{h}, x_{r}\right]\right)$, and on the angular frequency $\omega$. If the imposed temperature distribution is known, the transfer matrix $\mathbf{T}_{\mathbf{T C}}$ can be obtained theoretically $[1,2,17]$, but it can also be obtained from experiments under various heating conditions [22].

\subsection{Derivation of the characteristic equation}

\subsubsection{Standing wave engines}

The case of a standing wave engine is first considered here. If the matrix $\mathbf{T}_{\mathbf{T C}}$ is known, the theoretical modelling of the complete device requires knowledge of the acoustic propagation through the components surrounding the thermoacoustic core. This can be realized by deriving the expressions of the reflected impedances $Z_{l, r}=$ $\tilde{p}\left(x_{l, r}\right) / \tilde{u}\left(x_{l, r}\right)$ at both sides of the thermoacoustic core. For instance, if a standing-wave device as the one depicted in Fig. 1(a) is considered, writing the lossy propagation of harmonic plane waves at angular frequency $\omega$ in the ducts of respective lengths $x_{l}$ and $L-x_{r}$ leads to the expressions of the reflected impedances

$$
\begin{aligned}
& Z_{l}=\frac{\widetilde{p}\left(x_{l}\right)}{\widetilde{u}\left(x_{l}\right)}=\frac{Z_{0}+j Z_{c} \tan \left(k x_{l}\right)}{1+j Z_{0} Z_{c}^{-1} \tan \left(k x_{l}\right)}, \\
& Z_{r}=\frac{\widetilde{p}\left(x_{r}\right)}{\widetilde{u}\left(x_{r}\right)}=\frac{Z_{L}-j Z_{c} \tan \left(k\left(L-x_{r}\right)\right)}{1-j Z_{L} Z_{c}^{-1} \tan \left(k\left(L-x_{r}\right)\right)},
\end{aligned}
$$

where

$$
k=\frac{\omega}{c_{0}} \sqrt{\frac{1+(\gamma-1) f_{\kappa}}{1-f_{\nu}}}
$$


and

$$
Z_{c}=\frac{\rho_{0} c_{0}}{S} \frac{1}{\sqrt{\left(1-f_{\nu}\right)\left(1+(\gamma-1) f_{\kappa}\right)}}
$$

are the complex wave number and the characteristic impedance of the duct, respectively. In Eqs. (5) and (6), $\rho_{0}$ is the fluid density at room temperature, $c_{0}$ is the adiabatic sound speed, $\gamma$ is the specific heat ratio of the fluid, $S$ is the duct cross-section, and the functions $f_{\kappa}$ and $f_{\nu}$ characterize the thermal and viscous coupling between the oscillating fluid and the duct walls [2, 23]. In Eqs. (3) and (4), $Z_{0}$ and $Z_{L}$ stand for the acoustic impedances at positions $x=0$ and $x=L$, respectively. They can be, for instance, the radiation impedance of an open pipe [24], the infinite impedance of a rigid wall, or the acoustic impedance of an electrodynamic alternator, depending on the configuration of the standing-wave engine. Finally, combining Eqs. (3) and (4) with Eq. (2) and solving the associated system of two equations leads to the equation

$$
Z_{l} \mathbf{T}_{\mathbf{p p}}+\mathbf{T}_{\mathbf{p u}}-Z_{l} Z_{r} \mathbf{T}_{\mathbf{u p}}-Z_{r} \mathbf{T}_{\mathbf{u u}}=0
$$

Eq. (7) is the characteristic equation which accounts for both the processes operating through the thermoacoustic core and the dissipative/reactive processes operating in its surrounding components. This equation must be satisfied to describe the complete device properly.

\subsubsection{Travelling wave engines}

If the case of a travelling wave engine is now considered, and if the matrix $\mathbf{T}_{\mathbf{T C}}$ is known, it is also possible to derive a characteristic equation similar to Eq. (7). This implies to describe acoustic propagation at both sides of the thermoacoustic core. The basic idea is to make one loop in the device - each of the individual components being characterized by its transfer matrix - so that after one loop, the characteristic equation will ensure that one arrives at the same starting point. More precisely, there exists on the first hand the equation characterizing the propagation through the thermoacoustic core, Eq. (2), and on the other hand, it is possible to obtain an additional relation between the acoustic pressure and acoustic volume velocity at both sides of the thermoacoustic core by means of the total transfer matrix $\mathbf{T}_{\text {sur }}$ of the components surrounding the 
thermoacoustic core:

$$
\left(\begin{array}{c}
\widetilde{p}\left(x_{l}\right) \\
\widetilde{u}\left(x_{l}\right)
\end{array}\right)=\mathbf{T}_{\text {sur }} \quad \times\left(\begin{array}{c}
\widetilde{p}\left(x_{r}\right) \\
\widetilde{u}\left(x_{r}\right)
\end{array}\right) .
$$

For instance, if the particular geometry of Fig. 1-(b) is considered, and if the effects of the loop curvature are neglected, the matrix $\mathbf{T}_{\text {sur }}$ can be written as

$$
\mathbf{T}_{\text {sur }}=\mathbf{T}_{\mathbf{l}} \times \mathbf{T}_{\text {load }} \times \mathbf{T}_{\mathbf{r}},
$$

where the matrices

$$
\mathbf{T}_{\mathbf{l , \mathbf { r }}}=\left(\begin{array}{cc}
\cos \left(k d_{l, r}\right) & j Z_{c} \sin \left(k d_{l, r}\right) \\
j Z_{c}^{-1} \sin \left(k d_{l, r}\right) & \cos \left(k d_{l, r}\right)
\end{array}\right)
$$

characterize lossy propagation through the ducts of respective lengths $d_{l}=x_{l}$ and $d_{r}=L-x_{r}(L$ is the unwrapped length of the closed-loop), and where the matrix

$$
\mathbf{T}_{\text {load }}=\left(\begin{array}{cc}
1 & 0 \\
-Y_{\text {load }} & 1
\end{array}\right)
$$

accounts for the effect of the secondary acoustic load, by means of its reflected acoustic admittance $Y_{\text {load }}$ (this acoustic load can be a secondary acoustic resonator [20], an electrodynamic alternator [25] or any other component characterized by its reflected admittance $\left.Y_{\text {load }}\right)$.

Finally, combining Eq. (8) with Eq. (2) leads to the following characteristic equation:

$$
\operatorname{det}\left(\mathbf{T}_{\mathbf{T C}} \times \mathbf{T}_{\text {sur }}-\mathbf{I}_{\mathbf{2}}\right)=0,
$$

where $\mathbf{I}_{\mathbf{2}}$ stands for the identity matrix $2 \times 2$, and $\operatorname{det}()$ denotes the determinant of a matrix.

\subsection{Determination of the complex frequency}

The proper description of the thermoacoustic device requires to satisfy the corresponding characteristic equation

$$
f\left(\omega, T_{m}\right)=0
$$


where the function $f$ corresponds to the left-hand-side of Eq. (7) or Eq. (12), depending on the system under consideration. It is important to point out that all of the above equations are derived in the frequency domain, and due to this, it is implicitly assumed from Eq. (1) that the thermoacoustic system operates on steady state : this means that the angular frequency $\omega$ is purely real. In fact, the only condition for which Eq. (13) can be satisfied is that the temperature distribution $T_{m}(x)$ is fixed in such a way that there exists a value of the angular frequency $\omega$ which cancels the function $f$. Under such a condition the acoustic waves are neither amplified nor attenuated, and since nonlinear effects saturating the wave amplitude growth are not considered here, the solutions $\omega$ and $T_{m}$ correspond to the threshold of thermoacoustic instability. However, as it will be proposed in the following, one may describe from Eq. (13) the wave amplitude growth occuring after the onset of thermoacoustic instability under the quasi-steady state assumption. To do this, let the angular frequency be allowed to have an imaginary part $\epsilon_{g}$ :

$$
\omega=\Omega+j \epsilon_{g}
$$

so that the acoustic pressure

$$
p(x, t)=\Re\left(\widetilde{p}(x) e^{-j \omega t}\right)=e^{\epsilon_{g} t} \Re\left(\widetilde{p}(x) e^{-j \Omega t}\right) .
$$

is assumed to oscillate at frequency $\Omega=\Re(\omega)$, while the attenuation/growth of the sound wave is characterized by the thermoacoustic amplification coefficient $\epsilon_{g}$. However, $\epsilon_{g}$ is assumed to be small compared to the real part $\Omega$ of angular frequency, which means that the amplitude of the wave varies slowly at the time scale of few acoustic periods, diminishing or growing depending on the sign of $\epsilon_{g}$, in as much as the temperature distribution $T_{m}(x)$ stays constant at the scale of a few acoustic periods.

Under this quasi-steady state assumption, $\epsilon_{g}<<\Omega$, and for a constant temperature distribution $T_{m}$ (at the time-scale of a few acoustic periods) it is possible to solve Eq. (13) using conventional numerical methods, and to find a couple $\left(\Omega, \epsilon_{g}\right)$ which characterizes both the frequency of acoustic oscillations and the wave amplitude growth/attenuation. The advantages of this formulation are that it is well-suited for the prediction of the onset of thermoacoustic instability (as will be shown in the next section) but also more generally, as will be discussed 
in section 4, for the prediction of the engine's efficiency, provided that the nonlinear effects operating after the onset are properly described.

\section{Determination of the threshold of thermoacoustic instability}

The theoretical modelling presented in section 2 can be used at first to determine the marginal stability conditions of thermoacoustic devices, i.e. to find the purely real angular frequency $\omega=\Omega$ and the temperature distribution $T_{m}$ for which Eq. (13) is satisfied. However, before illustrating this with two particular examples, two remarks need to be formulated.

Firstly, the model of section 2 is actually incomplete since the heat transport within the thermoacoustic core needs to be described. Though it is well-established that thermoacoustic amplification depends significantly on the details of the temperature distribution, we will assume here for simplicity that a linear temperature distribution is imposed along the thermoacoustic core. In the absence of an appropriate description of heat transfer through the thermoacoustic core, the effect of heating will thus be represented by the temperature ratio $T_{h} / T_{c}$, where $T_{h}$ refers to the hot temperature at position $x_{h}$ and $T_{c}=300 \mathrm{~K}$ is the room temperature. In this paper, the transfer matrix $\mathbf{T}_{\mathbf{T C}}\left(\omega, T_{h} / T_{c}\right)$ of the thermoacoustic core is obtained from the transformation of the well-known differential wave equation of thermoacoustics [1, 2] into an equivalent Volterra integral equation of the second kind (see refs. $[10,17]$ for more details).

Secondly, in most of thermoacoustic devices the self-sustained oscillations are generated at a frequency which corresponds to the lower order acoustic mode of the system, but since some of the components of the device consist of a duct of finite length, Eq. (13) has actually an infinite number of solutions under the plane wave assumption (under some circumstances, higher order acoustic modes may even become more unstable than the first one, e.g. in refs $[18,26])$. Since conventional numerical methods for the solving of Eq. (13) should converge to a single solution, it is required to define an appropriate initial condition in the numerical scheme in order that the algorithm converges to the desired solution. 
In practice, the temperature ratio $T_{h} / T_{c}$ is fixed, acting as a parameter, and the characteristic equation (13) is solved using an iterative Newton-Raphson method, which is suitable for finding the roots of a complex function of a complex variable [27]. The solution of (13) is found using the following recurrence relation :

$$
\omega_{k+1}=\omega_{k}-\frac{f\left(\omega_{k}\right)}{f^{\prime}\left(\omega_{k}\right)}
$$

where the first derivative $f^{\prime}\left(\omega_{k}\right)$ is calculated using a simple first order finite difference

$$
f^{\prime}\left(\omega_{k}\right)=\left[\frac{d f}{d \omega}\right]_{\omega=\omega_{k}}=\frac{f\left(\omega_{k}+\Delta \omega_{k}\right)-f\left(\omega_{k}\right)}{\Delta \omega_{k}} .
$$

For our calculations, the step of finite difference is fixed to $\Delta \omega_{k}=1.10^{-3} \omega_{k}$. In order to avoid the divergence of the method, the initial value $\omega_{0}$ is fixed to the angular frequency of a resonant mode of the complete device. In the case of resonant modes resulting from a complicated coupling between different elements (see for instance the loop engine presented in Sect. 3.2), this initial angular frequency is determined graphically by plotting the modulus $|f(\Omega)|$ and by pointing at a local minimum of the function. The initial value for $\epsilon_{g}$ is fixed to $\epsilon_{g}=0$. The iterative computation is stopped when a sufficient accuracy $e$ is obtained on the solution, i.e. when

$$
\left|\omega_{k+1}-\omega_{k}\right|=\left|\frac{f\left(\omega_{k}\right)}{f^{\prime}\left(\omega_{k}\right)}\right| \leq \frac{e}{10}
$$

with $e=10^{-8}$ in our case. With this computation process, it is then possible to calculate $\omega=\Omega+j \epsilon_{g}$ as a function of the temperature $T_{h}$. The threshold of thermoacoustic instability then corresponds to the hot temperature $T_{h}$ for which $\epsilon_{g}=0$ : it is determined by means of a zero-finding method acting on the function $\epsilon_{g}\left(T_{h}\right)$.

\subsection{Standing wave engine}

As a basic illustration of the applicability of the model presented in Sect. 2, the case of a standing-wave engine closed at both ends is studied. A schematic drawing of this engine is shown in Fig. 1(a) : two straight cylindrical waveguides of respective lengths $d_{l}=x_{l}$ and $d_{r}=L-x_{r}$ are connected to the thermoacoustic core. Assuming that the thermoacoustic engine is closed at both ends, i.e. $Z_{0}=Z_{L}=\infty$, the expressions of the reflected 
impedances

$$
\begin{aligned}
& Z_{l}=-j Z_{c} \cot \left(k d_{l}\right), \\
& Z_{r}=j Z_{c} \cot \left(k d_{r}\right),
\end{aligned}
$$

are introduced into Eq. (7) to compute the angular frequency of acoustic oscillations $\Omega$ and the thermoacoustic amplification coefficient $\epsilon_{g}$ in functions of the hot temperature $T_{h}$. In this example, the total length of the engine is fixed to $L=1 \mathrm{~m}$, while the lengths of the stack and the thermal buffer tube are fixed to $l_{s}=l_{w}=5$ $\mathrm{cm}$. Air at atmospheric pressure is used as a working fluid, and the stack is modelized as an assembly of 0.5 mm-spaced parallel plates (50 $\mu \mathrm{m}$ in thickness).

Figure 2 presents the angular frequency $\Omega$ and the corresponding amplification coefficient $\epsilon_{g}$ as functions of the temperature ratio $T_{n}=\frac{T_{h}}{T_{c}}$ for three different locations of the stack in the resonator $: x_{h}=\frac{L}{4}(\ldots), x_{h}=\frac{L}{2}$ $(--)$ and $x_{h}=\frac{3 L}{4}(-)$. In Fig. 2, we focus only on the first eigen mode of the half-wavelength resonator and $\Omega$ remains close to the angular frequency $\Omega_{r}=\frac{\pi c_{0}}{L}$. For the two positions $x_{h}=\frac{L}{4}$ and $x_{h}=\frac{L}{2}$, when $T_{n}>1$, the amplification coefficient $\epsilon_{g}$ is negative and is continuously decreasing, meaning that the stack has a damping effect for these positions. By contrast, for $x_{h}=\frac{3 L}{4}$, the onset condition $\epsilon_{g}=0$ is found for a temperature ratio $T_{0} \simeq 1.5$. It can also be noticed that $\epsilon_{g}<0$ for $T_{n}<T_{0}$ (damping) and $\epsilon_{g}>0$ for $T_{n}>T_{0}$ (amplification). These conclusions are well-known for this configuration of engine [2], in which the thermoacoustic amplification only operates when the temperature gradient has the same sign as the acoustic pressure gradient.

It is worth noting that the angular frequency $\Omega$ is searched close to a resonant frequency of the device. It is thus possible to calculate $\Omega$ and $\epsilon_{g}$ for different modes. Figure 3 shows the results for $\Omega$ and $\epsilon_{g}$ as functions of the position $x_{h}$ of the stack in the resonator, for the three first resonant modes of the half-wavelength tube. The temperature is fixed to $T_{n}=4$, which is potentially sufficient for the onset of any of the three modes. It appears that $\epsilon_{g}$ can be positive for some values of $x_{h}$, depending on the mode under consideration, meaning that there exists particular positions for the stack, favourable to the onset of one or several modes. The comparison between Fig. 3 and the spatial distribution of the acoustic pressure fields in a half-wavelength resonator is quite 
direct and confirms that these favourable positions are those for which the temperature gradient has the same sign as the acoustic pressure gradient.

If the stack is near enough to the rigid wall on the right, then the three first modes can potentially become unstable. Figure 4 shows the angular frequency $\Omega$ and the amplification coefficient $\epsilon_{g}$ as functions of the temperature ratio $T_{n}$ for $\frac{x_{h}}{L}=0.9$ and for the three first modes. The higher the mode is, the higher the onset threshold temperature $T_{0}$ is. It is also interesting to observe that if $T_{n}$ was sufficiently large and led to the onset of the three modes, the frequencies of the three instabilities would be incommensurate, which could lead to complex quasiperiodic and chaotic behaviours of the system [28].

\subsection{Travelling wave engine}

In this section, the model is applied to a travelling wave loop engine, as schematically drawn in Fig. 1-(b). A double electrodynamic alternator is acting as the secondary acoustic load. Table 1 lists all the parameters introduced in the model.

In the previous section, it has been demonstrated that the choice of the frequency in the vicinity of which the onset frequency is computed is important. As it is illustrated in Fig. 5, when the resonant modes result from a complicated coupling between different elements, this particular frequency can be determined graphically by plotting the modulus $|f(\Omega)|$ and by pointing at a local minimum of the function. It is then possible to study the onset conditions of the thermoacoustic instability close to the first resonance of the loop, but also for a lower frequency, near the mechanical resonance of the alternator.

In Fig. 6, the amplification coefficient $\epsilon_{g}$ is plotted as a function of the temperature ratio $T_{n}$ for these two different modes. When the loop engine is not coupled with a secondary acoustic load (see dotted lines in Fig. 6), the onset of the thermoacoustic instability is found for a temperature ratio $T_{0} \simeq 2.4$ and the corresponding acoustic wavelength is close to the unwrapped length of the loop. Introducing the alternator as a secondary acoustic load does not impact significantly the onset conditions (see dashed lines in Fig. 6) : a small increase 


\begin{tabular}{|c|c|}
\hline \multicolumn{2}{|l|}{ Loop } \\
\hline Total length $L$ & $1 m$ \\
\hline Inner radius $R_{w}$ & $1 \mathrm{~cm}$ \\
\hline Location of the hot exchanger $x_{h}$ & $0.9 m$ \\
\hline Length of the regenerator $l_{s}$ & $4 \mathrm{~cm}$ \\
\hline Length of the thermal buffer tube $l_{w}$ & $8 \mathrm{~cm}$ \\
\hline \multicolumn{2}{|l|}{ Regenerator } \\
\hline Semi-width $R_{s}$ & $40 \mu m$ \\
\hline Porosity $\Phi$ & 0.75 \\
\hline \multicolumn{2}{|l|}{ Fluid (Helium) } \\
\hline Room temperature $T_{c}$ & $300 K$ \\
\hline Static pressure $P_{0}$ & $3 M P a$ \\
\hline \multicolumn{2}{|l|}{ Electrodynamic Alternators } \\
\hline Radius of the piston $R_{a l t}$ & $2 \mathrm{~cm}$ \\
\hline Moving mass $M_{m}$ & $0.2 \mathrm{~kg}$ \\
\hline Mechanical losses $R_{m}$ & $2 N . s . m^{-1}$ \\
\hline Stiffness of the suspensions $K_{m}$ & $5.10^{4}$ N. $m^{-1}$ \\
\hline $\mathrm{Bl}$ product & $20 N \cdot A^{-1}$ \\
\hline Coil electrical resistance $R_{e}$ & $6 \Omega$ \\
\hline Coil inductance $L_{e}$ & $50 m H$ \\
\hline \multicolumn{2}{|l|}{ Back Cavity (cylinder) } \\
\hline Inner radius $R_{c a v}$ & $4 \mathrm{~cm}$ \\
\hline Length $l_{\text {cav }}$ & $6 \mathrm{~cm}$ \\
\hline
\end{tabular}

Table 1: General characteristics of the travelling wave engine computed with the model. 
of the onset temperature is observed, due to additionnal losses in the alternator. However, it appears that the onset temperature for the coupled mode (continuous lines in Fig. 6) is lower than the one corresponding to the first mode of the loop. This behaviour is usually verified in practice, as this type of engine has generally an operating point close to the resonance of the alternator [25].

\subsection{Comparison with experimental results}

In 1998, Yazaki et al. studied a closed-loop thermoacoustic device and measured the stability curves as functions of the ratio $\left(r / \delta_{\kappa}\right)^{2}$, together with the acoustic work flow using LDV [18]. They notably observed that the frequency of the sound wave amplified by thermoacoustic effect was, surprisingly, close to the one of the second mode of the loop $(\lambda=L / 2)$ but not the first mode $(\lambda=L)$. In addition, they also investigated the same thermoacoustic device acting as a "standing-wave" engine by blocking the loop with a rigid partition : they observed the threshold of the fourth standing-wave mode (for which $\lambda=L / 2$ ).

In order to evaluate the consistency of our model, the thermoacoustic engine built by Yazaki et al. in 1998 has been considered and the stability curves have been calculated by varying the static pressure $P_{0}$ inside the engine. The onset temperature ratio $T_{0}$ is presented in Fig. 7 as a function of the square of the ratio $r / \delta_{\kappa}$. The results obtained by Yazaki et al. [18] for both configurations ( $\circ$ : annular device, • : straight device) are compared with the theoretical ones (straight lines), when the oscillating frequency is searched close to the frequency of the mode $\lambda=L / 2$. This frequency corresponds to the $2^{\text {nd }}$ mode of the annular device (called "TW" in Fig. 7) and to the $4^{\text {th }}$ mode of the straight device (called "SW" in Fig. 7). The model reproduces quite well the left branches of the stability curves, and it predicts an optimal ratio $r / \delta_{\kappa}$ close to the experimental one. For larger $r / \delta_{\kappa}$, the onset temperature ratio is less important when computed with the model. The differences between the model and the measurements realized by Yazaki et al. may be explained with the following statements. Firstly, the temperature distributions are supposed to be linear along the stack and the thermal buffer tube. Secondly, some parameters in our model, such as those of the heat exchangers, the length of the thermal buffer 
tube or the length of the glass pipe used for LDV, were fixed arbitrarily because they were unknown (all the parameters used for the design are reported in Table 2).

The model may also be used to investigate the typical mode selection observed by Yazaki et al. In his experiments [18], Yazaki replaced one part of the loop with a glass pipe of smaller inner radius used for velocity measurements by LDV. The resulting cross-sectional area constriction is not large (see Table 2), but we stated the fact that this constriction would be responsible for the mode selection, by "killing" the first mode of the loop. Indeed, when no constriction is introduced in the model, the first mode $(\lambda=L$, dashed line in Fig. 7$)$ naturally becomes unstable for lower temperature ratios than the second mode (dotted line). But when the cross-sectionnal area constriction is added, the first mode of the loop may theoretically become unstable for much larger, physically inconsistent, temperature ratios (e.g. $T_{0} \simeq 20$ for $\left(r / \delta_{\kappa}\right)^{2} \simeq 2.6$ ), and one can reasonably say that the onset is impossible. To conclude, the thermoacoustic engine built by Yazaki is not favourable to the onset of the first mode of the loop, due to this cross-sectionnal area constriction.

\section{About the applicability of the model for transient regimes}

As shown in Sect. 3, the model presented in Sect. 2 can be used for the determination of the onset conditions of the thermoacoustic instability, but another advantage of this model is that it can also be used for the theoretical study of the transient regime leading to steady-state sound.

If the accurate account of various nonlinear effects and the proper description of heat transfer through the thermoacoustic core are discarded in the context of this study, it is actually quite direct to propose the basic formulation which is necessary to compute the transient process of wave amplitude growth and saturation. On the first hand, let's assume that it is possible to describe heat transfer through the thermoacoustic core by means of the following differential equation:

$$
\partial_{t} T(x, t)=g(T(x, t), \ldots),
$$

where $\partial_{t}$ stands for partial time derivative, and where $T(x, t)$ refers to the time-dependent, cross-sectional 


\begin{tabular}{|c|c|c|}
\hline & $\begin{array}{c}\text { Yazaki et al. } \\
{[18]}\end{array}$ & Model \\
\hline Total length & $2.58 m$ & $2.58 m$ \\
\hline Inner radius $R_{1}$ & $20.1 \mathrm{~mm}$ & $20.1 \mathrm{~mm}$ \\
\hline \multicolumn{3}{|l|}{ Length of } \\
\hline the glass pipe & unknown & $0.94 m$ \\
\hline \multicolumn{3}{|l|}{ Inner radius of } \\
\hline the glass pipe $R_{2}$ & $18.5 \mathrm{~mm}$ & $18.5 \mathrm{~mm}$ \\
\hline \multicolumn{3}{|l|}{ Location of the } \\
\hline center of the stack & $0.5 m$ & $0.5 m$ \\
\hline Length of the stack & $4 \mathrm{~cm}$ & $4 \mathrm{~cm}$ \\
\hline \multicolumn{3}{|l|}{ Length of the thermal } \\
\hline buffer tube & unknown & $20 \mathrm{~cm}$ \\
\hline \multicolumn{3}{|l|}{ Length of the ambient } \\
\hline heat exchanger & unknown & $2 \mathrm{~cm}$ \\
\hline \multicolumn{3}{|l|}{ Length of the hot } \\
\hline heat exchanger & unknown & $2 \mathrm{~cm}$ \\
\hline \multicolumn{3}{|l|}{ Semi-width of } \\
\hline one stack pore & $0.44 m m$ & $0.44 m m$ \\
\hline Porosity of the stack & 0.72 & 0.72 \\
\hline \multicolumn{3}{|l|}{ Semi-width of one } \\
\hline exchanger pore & unknown & $2 \times 0.4 \mathrm{~mm}$ \\
\hline Porosity of exchangers & unknown & 0.88 \\
\hline Room temperature $T_{c}$ & unknown & $293 K$ \\
\hline
\end{tabular}

Table 2: Characteristics of the thermoacoustic engine designed by Yazaki et al. [18] 
average temperature distribution along the thermoacoustic core. In this equation the function $g$ is supposed to account for the heat transfer processes (diffusion, convection, radiation) controlling the temperature distribution, while the dots in the argument of the function $g$ refer to the geometrical and thermophysical parameters of the device under consideration. On the other hand, the thermoacoustic amplification operating in the thermoacoustic core is described with the ordinary differential equation:

$$
d_{t} p_{r m s}\left(x_{0}, t\right)=\epsilon_{g}(T(x, t)) p_{r m s}\left(x_{0}, t\right)
$$

where $d_{t}$ denotes time derivative, where $p_{r m s}\left(x_{0}, t\right)=\sqrt{\left\langle p^{2}\left(x_{0}, t\right)>\right.}$ is the root mean square amplitude of acoustic pressure oscillations at some position $x_{0}$ along the device $(<\cdots>$ denotes time averaging over an acoustic period), and where $\epsilon_{g}$ is the imaginary part of the complex frequency introduced in Sect. 2. Note that this equation is directly derived from Eq. (1) because $p_{r m s}\left(x_{0}, t+\Delta t\right)=e^{\epsilon_{g} \Delta t} p_{r m s}\left(x_{0}, t\right)$, and because it is assumed that $\epsilon_{g}<<\Omega$ and that during the time scale $\Delta t$ of a few acoustic periods, the variations of the temperature distribution are negligible, so that $\epsilon_{g}$ stays constant.

In order to compute the initial start-up of thermoacoustic oscillations up to the final stabilization of acoustic pressure amplitude, one must solve the set of coupled equations (21) and (22) with appropriate boundary and initial conditions, provided that the nonlinear effects saturating the wave amplitude growth are included in these equations. For instance, the effect of thermoacoustic heat pumping by the acoustic wave, which tends to reduce the temperature gradient externally imposed along the stack, can be included in Eq. (21) in the form of an acoustically (proportional to $p_{r m s}^{2}$ ) enhanced thermal conductivity [12, 29] of the stack . More generally, as in the case of the DeltaEC computer code, it is possible to account (in a necessary simplified way) for some of the nonlinear effects involved in the saturation process, such as minor losses at the edges of the stack [30], higher harmonics generation $[9,10,12]$, or heat convection due to acoustic streaming $[31,32,33]$.

As mentioned above, no further derivations are presented in this paper concerning the modeling of unsteady processes in thermoacoustic engines. This would indeed require to define the precise geometry of the device under consideration and the thermophysical properties of its components, to quantify properly each of the 
saturating processes, and also to perform comparisons with experimental data. We will defer such studies to a future publication. Our main goal here is to point out the advantages of the formulation proposed in this paper, which can be used to study unsteady processes occuring in thermoacoustic systems of complicated geometries. The transient regime which precedes the stabilization of acoustic pressure amplitude can exhibit complicated behaviours $[7,8,34,35]$, and it provides much more information than the only value of a steady state acoustic pressure. If one is able to reproduce such complicated dynamics using an appropriate simplified model, then one is able to get a better physical insight on the nonlinear processes which control the saturation of the thermoacoustic instability.

\section{Conclusion}

We presented a formulation for the description of the wave amplification in different kinds of thermoacoustic engines. The model is suitable for the determination of the threshold of thermoacoustic instability, and it can be coupled to the equations describing heat transfer through the thermoacoustic core in order to describe unsteady processes leading to steady-state sound in the frame of the weakly nonlinear theory. In the frame of the linear theory, this model captures some interesting properties of thermoacoustic engines, as the stack-location influence on the onset of the thermoacoustic instability or the selection of the unstable resonant mode of the device which can be amplified. As a complementary tool to the computer code DeltaEC or to direct numerical simulations, the proposed analytical model should prove useful for designing thermoacoustic engines and for investigating the nonlinear processes involved in these devices.

\section{Acknowledgments}

We would like to thank Pierrick Lotton for his useful advices concerning the manuscript. 


\section{References}

[1] N. Rott. "Thermoacoustics". Advances in Applied Mechanics, 20:135-175, 1980.

[2] G.W. Swift. Thermoacoustics - A unifying perspective for some engines and refrigerators. Acoustical Society of America, Melville, NY, 2002.

[3] S.L. Garrett. Resource letter: TA-1: Thermoacoustic engines and refrigerators. Am. J. Phys., 72(1):11-17, 2004.

[4] W.C. Ward, G.W. Swift, and J.P. Clark. Interactive analysis, design, and teaching for thermoacoustics using DeltaEC. J. Acoust. Soc. Am., 123(5):3546-3546, 2008.

[5] G.B. Chen and T. Jin. Experimental investigation on the onset and damping behavior of the oscillation in a thermoacoustic prime mover. Cryogenics, 39(10):843-846, 1999.

[6] T. Jin, C.S. Mao, and K. Tang. Characteristics study on the oscillation onset and damping of a travelingwave thermoacoustic prime mover. Journ. Zhejiang Univ. Science A, 9:944-949, 2008.

[7] G.W. Swift. Analysis and performance of a large thermoacoustic engine. J. Acoust. Soc. Am., 92(3):15511563, 1992.

[8] G. Penelet, V. Gusev, P. Lotton, and M. Bruneau. Nontrivial influence of acoustic streaming on the efficiency of annular thermoacoustic prime movers. Phys. Lett. A, 351(4-5):268-273, 2006.

[9] S. Karpov and A. Prosperetti. Nonlinear saturation of the thermoacoustic instability. J. Acoust. Soc. Am., 107(6):3130-3147, 2000.

[10] V. Gusev, H. Bailliet, P. Lotton, and M. Bruneau. Asymptotic theory of nonlinear acoustic waves in a thermoacoustic prime mover. Acust. Acta Acust., 86:25-38, 2000.

[11] A.T.A.M. de Waele. Basic treatment of onset conditions and transient effects in thermoacoustic Stirling engines. J. Sound Vib., 325(4-5):974-988, 2009. 
[12] G. Penelet, V. Gusev, P. Lotton, and M. Bruneau. Experimental and theoretical study of processes leading to steady-state sound in annular thermoacoustic engines. Phys. Rev. E, 72(1):016625, 2005.

[13] S. Karpov and A. Prosperetti. A nonlinear model of thermoacoustic devices. J. Acoust. Soc. Am., 112(4):1431-1444, 2002.

[14] M.F. Hamilton, Y.A. Ilinskii, and E.A. Zabolotskaya. Nonlinear two-dimensional model for thermoacoustic engines. J. Acoust. Soc. Am., 111(5):2076-2086, 2002.

[15] J.E. Parker, M.F. Hamilton, Y.A. Ilinskii, and E.A. Zabolotskaya. Use of complex frequency to analyze thermoacoustic engines. J. Acoust. Soc. Am., 112(5):2297-2297, 2002.

[16] Q. Tu, Q. Li, F. Wu and F. Z. Guo. Network model approach for calculating oscillating frequency of thermoacoustic prime mover. Cryogenics, 43:351-357, 2003.

[17] G. Penelet, S. Job, V. Gusev, P. Lotton, and M. Bruneau. Dependence of sound amplification on temperature distribution in annular thermoacoustic engines. Acust. Acta Acust., 91(3):567-577, 2005.

[18] T. Yazaki, A. Iwata, T. Maekawa, and A. Tominaga. Traveling wave thermoacoustic engine in a looped tube. Phys Rev. Lett., 81(15):3128-3131, 1998.

[19] P.H. Ceperley. A pistonless Stirling engine-The traveling wave heat engine. J. Acoust. Soc. Am., $66(5): 1508-1513,1979$.

[20] S. Backhaus and G.W. Swift. A thermoacoustic Stirling heat engine. Nature, 399:335-338, 1999.

[21] K.J. Bastyr and R.M. Keolian. High-frequency thermoacoustic-Stirling heat engine demonstration device. ARLO, 4(2):37-40, 2003.

[22] M. Guedra, G. Penelet, P. Lotton, and J.-P. Dalmont. Theoretical prediction of the onset of thermoacoustic instability from the experimental transfer matrix of a thermoacoustic core. J. Acoust. Soc. Am., 130:145152, 2011 
[23] W.P. Arnott, H.E. Bass, and R. Raspet. General formulation of thermoacoustics for stacks having arbitrarily shaped pore cross sections. J. Acoust. Soc. Am. 90(6):3228-3237, 1991.

[24] J.-P. Dalmont, C.J. Nederveen, and N. Joly. Radiation impedance of tubes with different flanges: numerical and experimental investigations. J. Sound Vib., 244(3):505-534, 2001.

[25] S. Backhaus, E. Tward, and M. Petach. Traveling-wave thermoacoustic electric generator. Appl. Phys. Lett., 85(6):1085-1087, 2004.

[26] T. Biwa, Y. Ueda, T. Yazaki, and U. Mizutani. Thermodynamical mode selection rule observed in thermoacoustic oscillations. EuroPhys. Lett., 60(3):363-368, 2002.

[27] Kincaid, D. and Cheney, W.. Numerical Analysis. Brooks/Cole, 1996

[28] T. Yazaki, S. Takashima, and F. Mizutani. Complex quasiperiodic and chaotic states observed in thermally induced oscillations of gas columns. Phys. Rev. Lett., 58(11):1108-1111, 1987.

[29] T. Yazaki, A. Tominaga, and Y. Narahara. Large heat transport due to spontaneous gas oscillation induced in a tube with steep temperature gradients. J. Heat Transfer, 105(4):889-894, 1983.

[30] R.S. Wakeland and R.M. Keolian. Measurements of the resistance of parallele-plate heat exchangers to oscillating flow at high amplitudes. J. Acoust. Soc. Am., 115(5):2071-2074, 2004.

[31] S. Boluriaan and P.J. Morris. Acoustic streaming: from Rayleigh to today. Intern. Journ. of Aeroacoustics, 2(3):255-292, 2003.

[32] V. Gusev, S. Job, H. Bailliet, P. Lotton, and M. Bruneau. Acoustic streaming in annular thermoacoustic prime-movers. J. Acoust. Soc. Am., 108(3):934-945, 2000.

[33] H. Bailliet, V. Gusev, R. Raspet, and R.A. Hiller. Acoustic streaming in closed thermoacoustic devices. J. Acoust. Soc. Am., 110(4):1808-1821, 2001. 
[34] G. Penelet, E. Gaviot, V. Gusev, P. Lotton and M. Bruneau. Experimental investigation of transient nonlinear phenomena in an annular thermoacoustic prime-mover: observation of a double-threshold effect. Cryogenics, 42(9):527-532, 2002.

[35] Z. Yu, A.J. Jaworski, and A.S. Abduljalil. Fishbone-like instability in a looped-tube thermoacoustic engine. J. Acoust. Soc. Am., 128(4):188-194, 2010. 

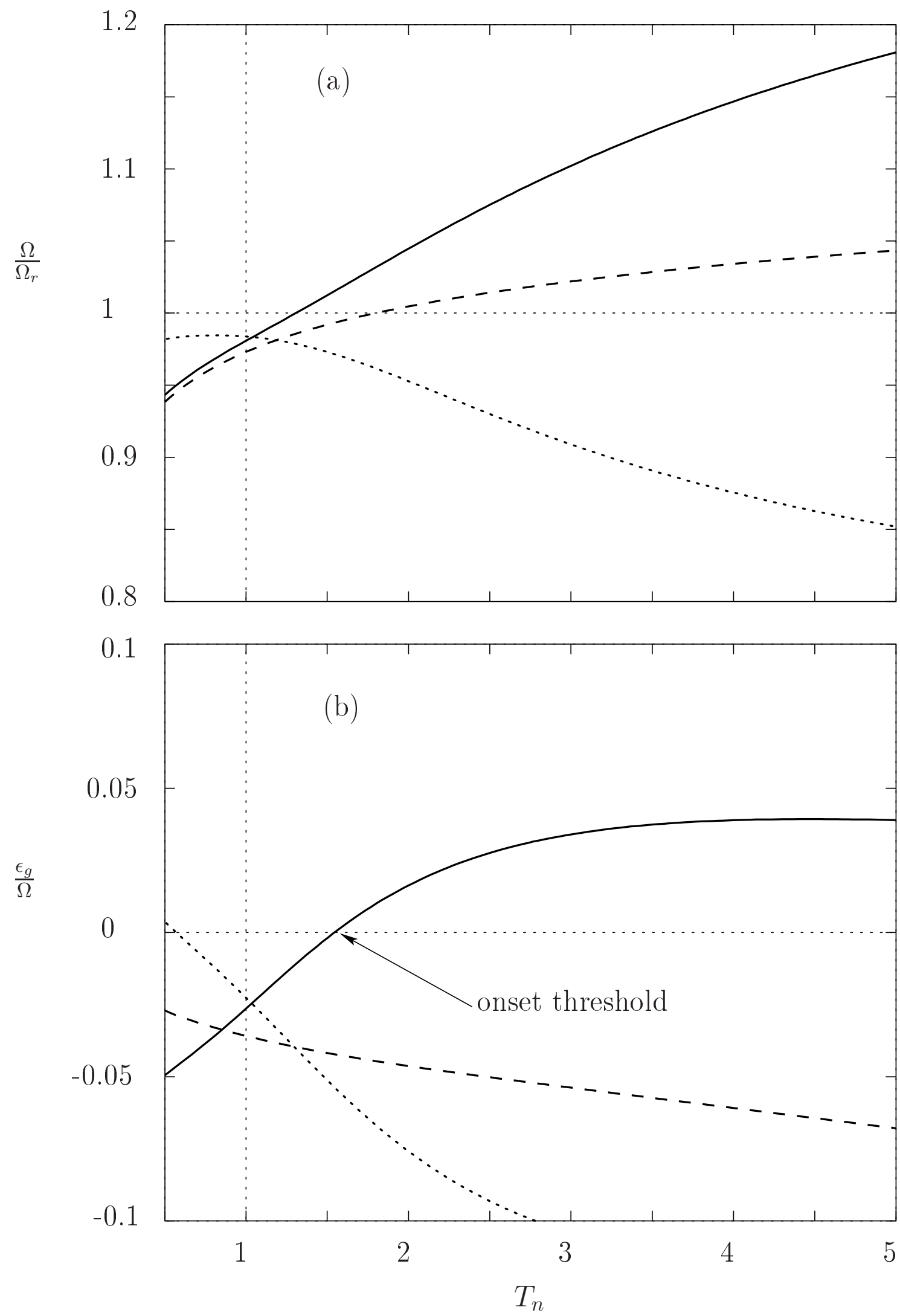

Figure 2: Normalized frequency $\frac{\Omega}{\Omega_{r}}$ (a) and amplification coefficient $\epsilon_{g}$ (b) as functions of the temperature ratio $T_{n}=\frac{T_{h}}{T_{c}}$ for three different locations of the stack in the resonator $: x_{h}=\frac{L}{4}(\ldots), x_{h}=\frac{L}{2}(--)$ and $x_{h}=\frac{3 L}{4}(-)$. For $x_{h}=\frac{3 L}{4}$, the onset threshold corresponds to $T_{n} \simeq 1.5$. 

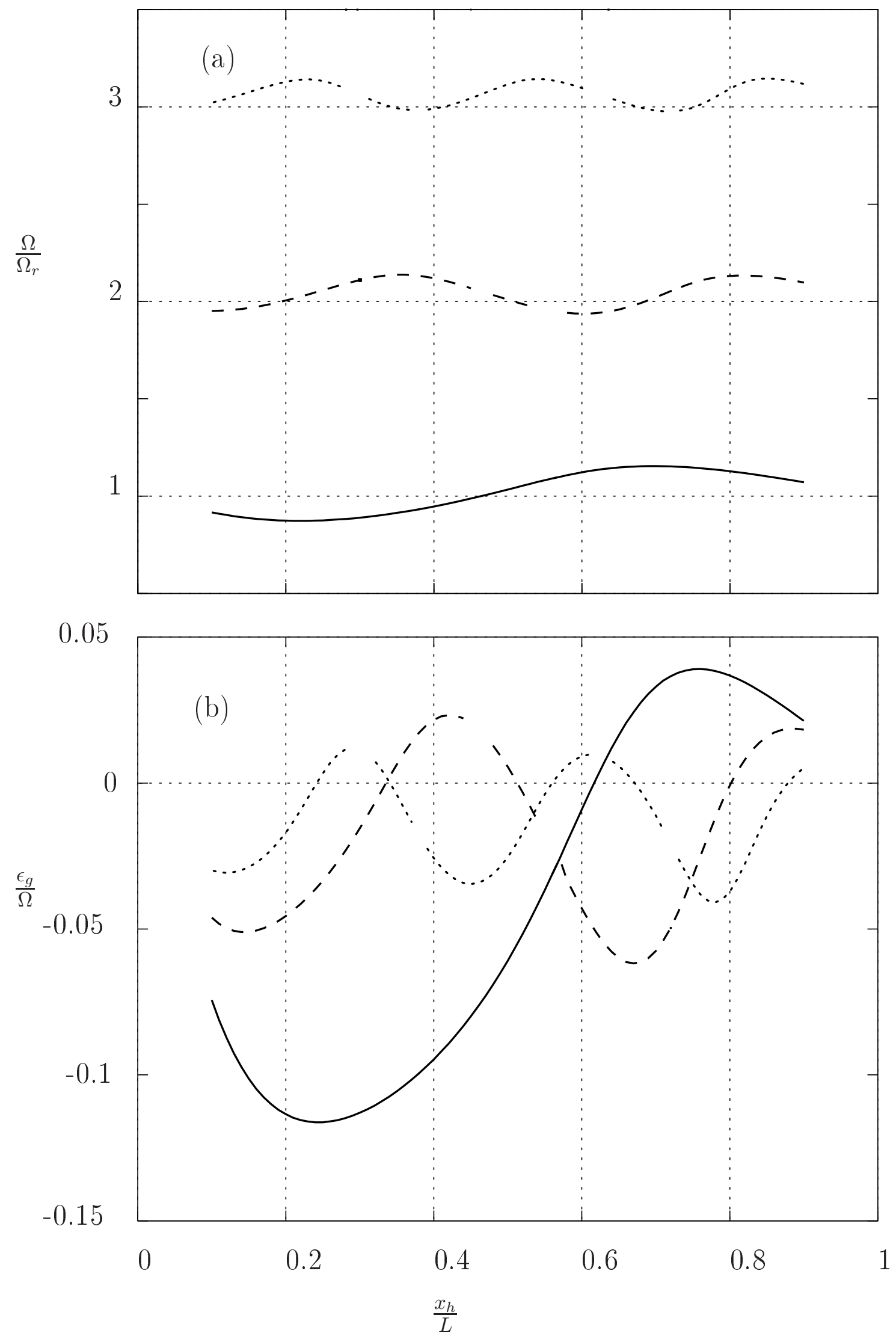

Figure 3: Normalized frequency $\frac{\Omega}{\Omega_{r}}$ (a) and amplification coefficient $\epsilon_{g}$ (b) as functions of the position of the stack $x_{h}$ in the resonator for the three first modes of the half-wavelength resonator : $\Omega_{r}=\frac{\pi c_{0}}{L}(-), 2 \Omega_{r}(--)$ and $3 \Omega_{r}(\ldots)$. Here, the temperature ratio is fixed to $\frac{T_{h}}{T_{c}}=4$. 

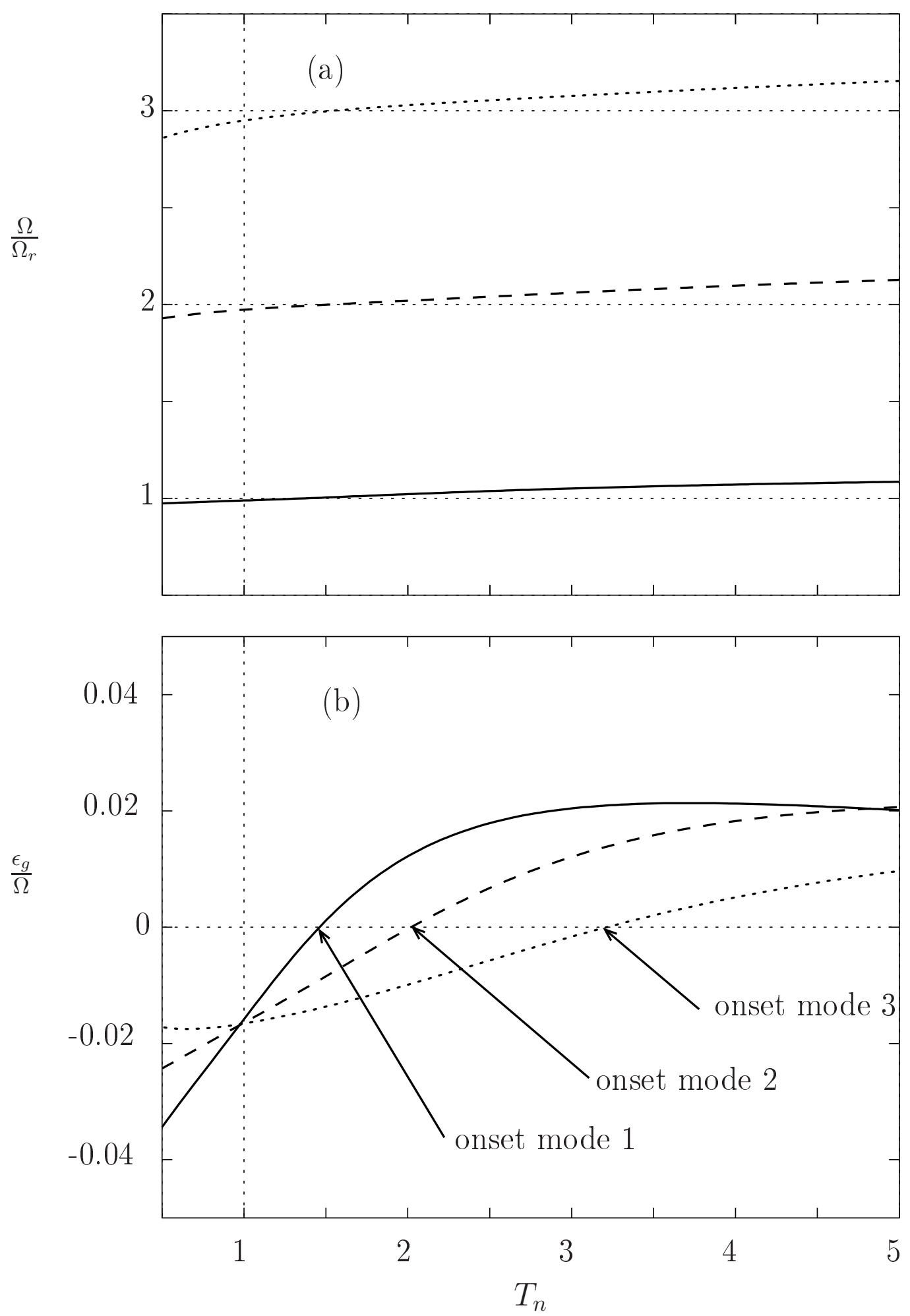

Figure 4: Normalized frequency $\frac{\Omega}{\Omega_{r}}$ (a) and amplification coefficient $\epsilon_{g}$ (b) as functions of the temperature ratio $T_{n}=\frac{T_{h}}{T_{c}}$ for the three first modes of the half-wavelength resonator. The location of the stack $x_{h}=0.9 L$ is chosen so that the onset of the three modes is achievable. 


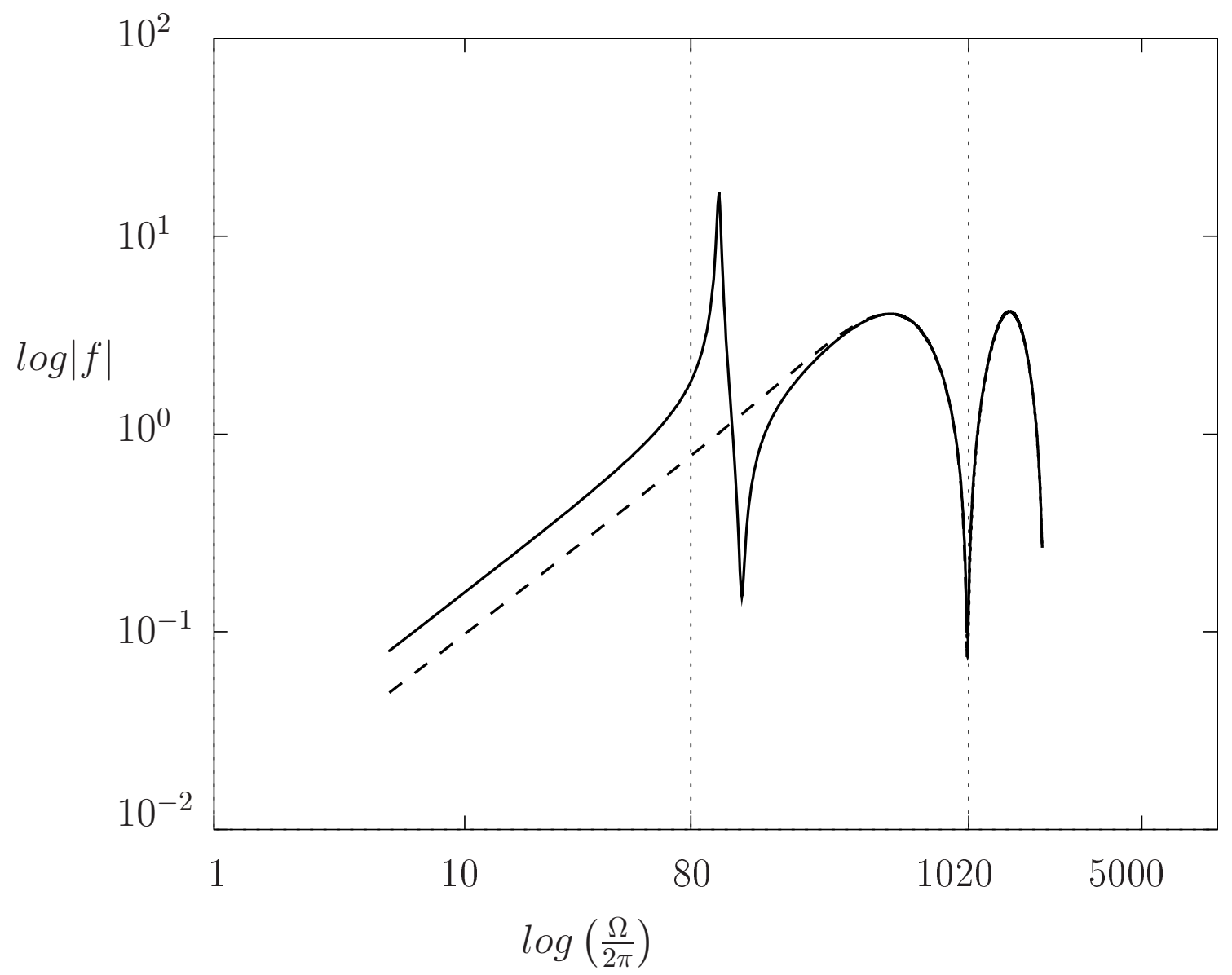

Figure 5: Modulus of the function $f\left(\Omega, T_{n}=1\right)$ computed for a closed-loop engine $(--)$ and for the closedloop engine coupled with the double alternator (-). The frequencies $80 \mathrm{~Hz}$ and $1020 \mathrm{~Hz}$ correspond to the mechanical resonance of the alternator and the first mode of the loop, respectively. 

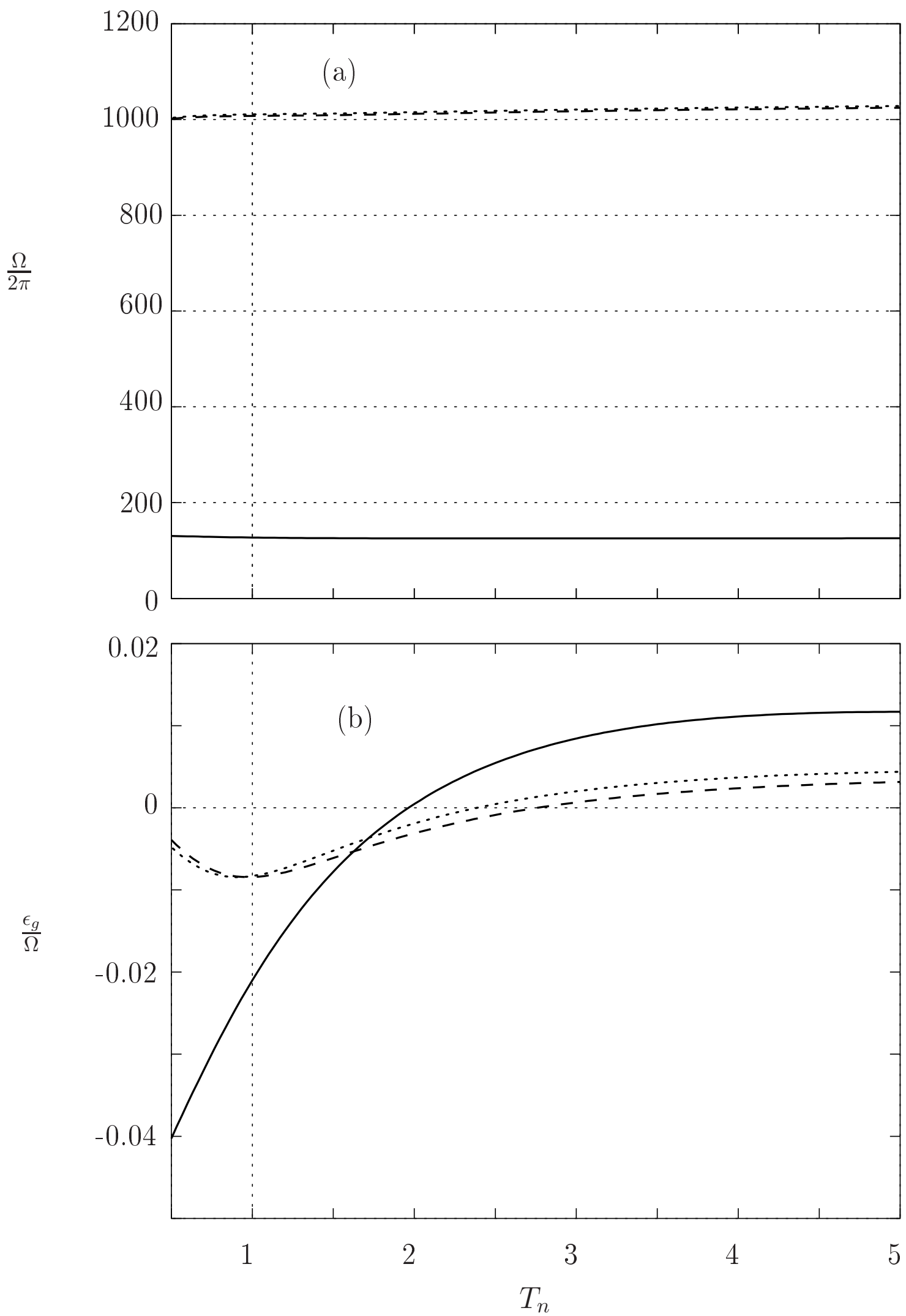

Figure 6: Frequency (a) and amplification coefficient (b) as functions of the temperature ratio $T_{n}=\frac{T_{h}}{T_{c}}$ for the first mode of the loop without alternator $(. .$.$) and for the two modes resulting from the coupling of the$ two elements : the mode close to the resonance of the alternator $(-)$ and the mode close to the first resonance of the loop $(--)$. 


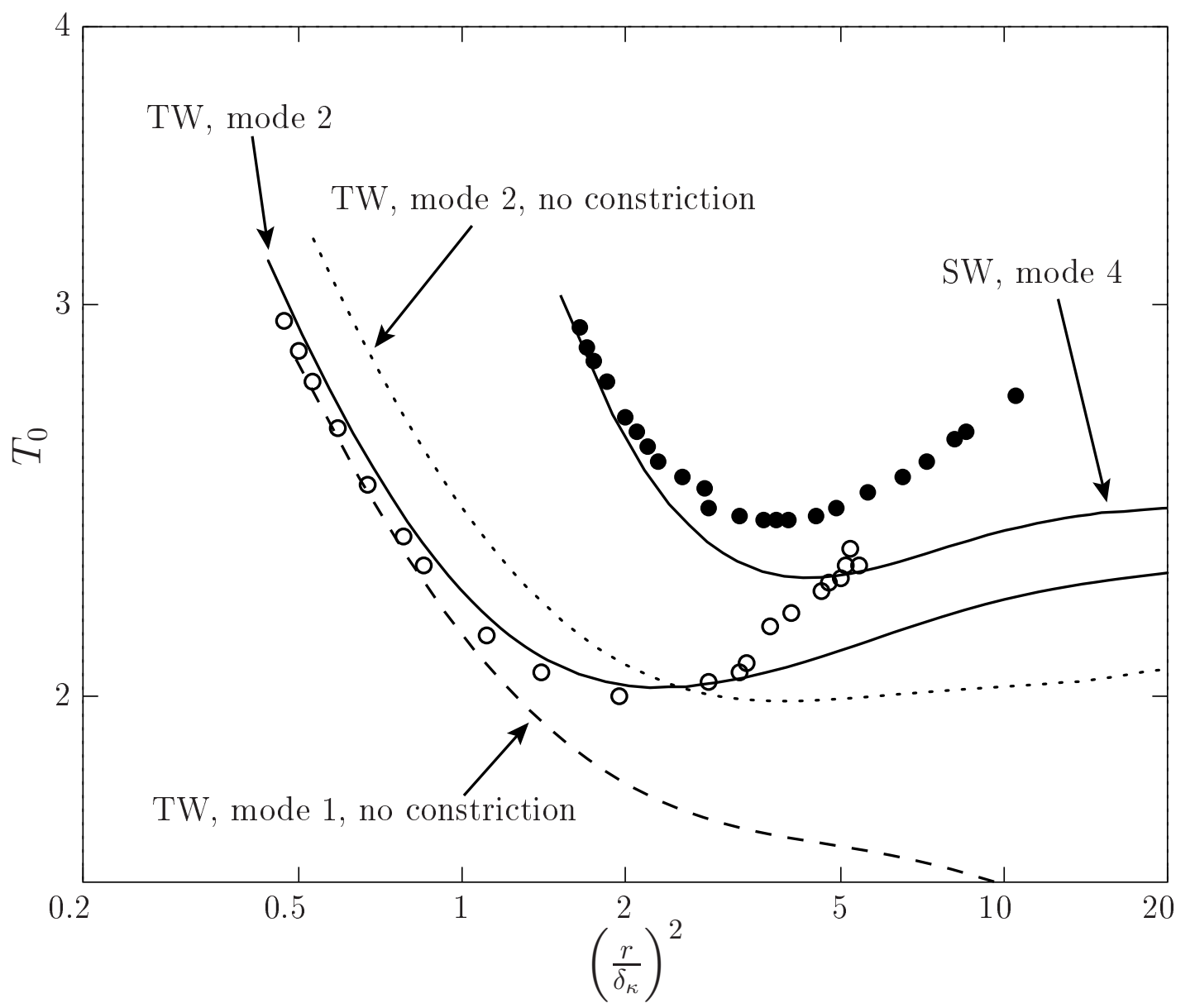

Figure 7: Stability curves for the second mode of the loop (o) and the fourth mode of the straight device $(\bullet)$ obtained by Yazaki et al. [18], compared with the theoretical ones (straight lines). In addition are plotted the stability curves for the first mode (dashed line) and the second mode (dotted line) of the loop when no cross-sectionnal area constriction is introduced in the model. 